\title{
ETHICAL ASPECTS OF THE USE OF INNOVATIVE INFORMATION TECHNOLOGIES IN CLINICAL TRIALS \\ Nikoleta Leventi ${ }^{1}$, Alexandrina Vodenitcharova ${ }^{2}$, Kristina Popova ${ }^{3}$
}

\begin{abstract}
:
A clinical trial, according to the WHO, "is any research study that prospectively assigns human participants or groups of humans to one or more health-related interventions to evaluate the effects on health outcomes. Interventions include but are not restricted to drugs, cells and other biological products, surgical procedures, radiological procedures, devices, behavioural treatments, process-of-care changes, preventive care, etc".

The application of innovative information technologies like artificial intelligence and big data analytics in clinical trial processes is a new challenge. Such systems are useful tools, and promise to enhance the healthcare management, and to optimize clinical outcomes and economic effectiveness. However, their use raises ethical and social issues.

In this direction, the European Commission in June 2018 set up the High Level Expert Group on AI, which offers guidance on a comprehensive framework for trustworthy AI. Trustworthy AI consists of three components, which should be met during the entire life cycle of the system: (1) it should be lawful, (2) it should be ethical, and (3) it should be robust.

In this article we used the focus group methodology to obtain information from experts about the ethical aspects raised when innovative information technologies like artificial intelligence and big data analytics are used in clinical trials. Feedback from the experts was also gathered regarding the usage of the proposed guidelines for trustworthy AI, as an evaluation tool for the particular case of clinical trials.
\end{abstract}

UDC Classification: 614.2, DOI: https://doi.org/10.12955/pmp.v1.100

Keywords: medical ethics, clinical trials, trustworthy AI.

\section{Introduction}

Clinical trials (CTs) are significant for the development of new methods in human treatment. According to the WHO (WHO, 2020) "a clinical trial is any research study that prospectively assigns human participants or groups of humans to one or more health-related interventions to evaluate the effects on health outcomes. Interventions include but are not restricted to drugs, cells and other biological products, surgical procedures, radiological procedures, devices, behavioural treatments, process-of-care changes, preventive care, etc". But, the path of new medicines is long, heavily regulated and controlled, and very expensive. To bring a new drug to the market takes on average 10 15 years and USD 1.5-2.0 billion. The clinical trial phases of the drug development cycle consume around half of this time and investment (Harrer et al., 2019). Meaning most of these extra costs are due to clinical trials. This is the reason to seek and introduce innovative technologies in clinical trials.

Innovative information technologies are widely used in medicine, implementing different systems including mobile telemedicine information systems for patients, medical doctors and other participants in the healthcare ecosystem (Patias \& Georgiev, 2017). Concrete innovative information technologies used in CTs include technologies such as artificial intelligence, big data analytics, blockchain, Internet of Things, virtual and augmented reality, simulations or gamification.

\section{Principles of medical ethics}

Medical ethics is the oldest professional ethics and is aimed at solving ethical problems that have arisen in the professional activity of doctors as well as other medical professionals. Traditional medical ethics begin with the Hippocratic Oath, a symbol of the uniqueness of medical practice and the oldest evidence of a code of ethics (Vodenitcharova \& Popova, 2019).

The four principles of biomedical ethics (Beauchamp \& Childress, 2013; Vodenitcharova \& Popova, 2019) are the principal of respect to the patient autonomy, beneficence, the principle of nonmaleficence, and the principle of justice:

\section{Principle of respect for autonomy}

The term autonomy means the individual's capacity for self-determination. Everyone can make dependent decisions and actions, and therefore the principle of patient autonomy is recognized as the

\footnotetext{
${ }^{1}$ Faculty of Public Health, Department of HTA, Medical University of Sofia, n.leventi@foz.mu-sofia.bg

${ }^{2}$ Faculty of Public Health, Department of Health Policy and Management, Medical University of Sofia, al.vodenicharova@abv.bg

${ }^{3}$ Faculty of Public Health, Department of Social Medicine, Medical University of Sofia, popova_kristina@yahoo.com
} 
opposite of the traditional approach in medicine, namely paternalism. For the patient to be autonomous, there must be two conditions:

$\bigcirc$ freedom i.e. independence of any control, and

- action - competence to be able to do this consciously.

2. Principle of beneficence

In the relationship between the physician and the patient, the healthcare professional has a moral obligation not to cause harm, but also to act for the benefit and contribute to the wellbeing of the patient. The mission of the physicians and other healthcare professionals is to help their patient by helping him or her to recover, or to help the community at large, for example with health prevention programs. The main goal of the doctor is to strive to make good and to protect the patient from risks and harms.

\section{Principle of non maleficence}

The principle of non-maleficence nature requires that no harm be caused intentionally. This principle is based on the maxim "Primum non nocere" which means, "First of all do no harm".

4. Principle of justice

The principle of justice refers in the context of distributive justice means to act impartially, honestly, and appropriately with respect to a person in a given situation, depending on what is due to him or her in the autonomy and in accordance with the goods, benefits and burdens of the society in relation to the accepted norms. Because some resources are scarce, they must be distributed fairly in society.

\section{Principles of bioethics}

In 1970 biochemist Van Rensselaer Potter coined a new word Bioethics. His aim was to focus on the lack of attention paid to the values due to the rapid advances in science (Apressyan et. al., 2008). Bioethics as newly defined discipline aims according to Potter to build a 'bridge' and connect 'facts' with 'values'. The word bioethics uses two Greek words. First, the word ßío (bios), life, represents the life facts and life sciences. Second, the word nं $\theta$ os (éthos), morals, refers to physicians', and other health care professionals', values, and duties, and the decisions, they make in their daily practice. The idea is that life facts contain values. Meaning for instance there are concrete conditions, which may cause pain or threaten the life of a patient or reduce somehow his well-being.

For physicians and health care professionals the promotion and implementation of values is their duty. And this is the origin of norms, namely the promotion of values. The general, widely described norms, we call them principles. In 2005 the UNESCO Universal Declaration on Bioethics and Human Rights (UNESCO, 2005) identified fifteen bioethical principles:

1. Human dignity and human rights;

2. Benefit and harm;

3. Autonomy and individual responsibility;

4. Consent;

5. Persons without the capacity to consent;

6. Respect for human vulnerability and personal integrity;

7. Privacy and confidentiality;

8. Equality, justice and equity;

9. Non-discrimination and non-stigmatization;

10. Respect for cultural diversity and pluralism;

11. Solidarity and cooperation;

12. Social responsibility and health;

13. Sharing of benefits;

14. Protecting future generations; and

15. Protection of the environment, the biosphere and biodiversity.

\section{Trustworthy Artificial Intelligence}

Combining issues related to innovative information technologies, and ethics, the European Commission (EC) in June 2018 set up a High-Level Expert Group on AI (AI HLEG) to propose guidelines for a comprehensive framework for robust AI (AI HLEG, 2018). The Guidelines aim to 
promote Trustworthy Artificial Intelligence, focusing on three main components, which should be met during the entire life cycle of the system, as trustworthy AI should be:

- lawful: meaning to comply with all applicable laws and regulations;

- ethical: meaning to guarantee compliance with the accepted ethical values, norms, and principles; and

- robust: meaning to cover both the technical and social perspectives, and avoid any unintentional harm, which even with good intentions, AI systems can cause.

For the achievement of Trustworthy AI, those three components are all necessary but not sufficient. They should all together work in harmony. In many cases they overlap, but as they develop, also the society develops and should endeavour to align them and avoid discrepancies.

The major ethical principles of respect for human autonomy, prevention of harm, fairness and explicability must be our guide. Any AI systems that we develop, deploy, and use must take into consideration and comply with those principles. Permanent attention should be paid to identifying and addressing potential tensions between those principles.

The proposed by AI HLEG framework aims to focus on the second (fostering and securing ethical AI) and third (fostering and securing robust AI) components. Trustworthy AI's first component (lawful $\mathrm{AI}$ ) is not cover, since it has to do with applicable laws and regulations. Finally, the proposed Guidelines is not a list of ethical principles, they are addressed to all stakeholders, and aim to provide guidance on how such principles can be used in practice.

Regarding the seven key requirements for Trustworthy AI the main advice is to ensure that the development, deployment and use of AI systems meet them, namely:

1. human agency and oversight,

2. technical robustness and safety,

3. privacy and data governance,

4. transparency,

5. diversity, non-discrimination and fairness,

6. environmental and societal well-being and

7. accountability.

In healthcare, medical professionals need to make clinical decisions, based on values and evidences. The obligation to uphold values is the source of norms. When such norms are pervasive and inclusive, they are called principles. However, with the introduction of innovative information technologies, the implementation of these principles itself represents a new challenge.

The aim of this paper is to demonstrate the ethical aspects that rise by the application of innovative information technology in CTs. Implementation of the proposed framework to promote Trustworthy $\mathrm{AI}$ is under consideration. Attempts are being made to extend the scope to technologies other than AI, but focused in the field of CTs.

\section{Methodology}

The study was conducted in November 2019. The methodology used in this study was based on a focus group discussion. The respondents participated voluntarily and anonymously. They were all experts from the Faculty of Public Health of the Medical University - Sofia, Bulgaria, and most of them members of the Bulgarian Unit of UNESCO Chair in Bioethics. The focus group consisted of six participants. The participants were asked for feedback through a focused questionnaire defining the discussion framework. A moderator led the focus group. An assistant moderator was used to help with recording and feedback reports preparation. A well-developed presentation of the European-level sample initiative, describing the topic was prepared for the purpose of the discussion. The participants were introduced to the topic and then were asked a few questions that play the role of a framework to support the discussion. The focus group session lasted about 90 minutes and included participants with interests related to CTs from different perspectives (such as, legislative, medical, marketing, etc.).

The purpose of the focus group discussion was to reflect the participants' views on the seven requirements for Trustworthy AI. The idea was to see the impact of the seven requirements in other innovative information technologies, except in the development, implementation and use of AI systems. Special focus was given in the case of clinical trials, and on the ethical challenges that arise. 
The questions for the discussion framework were focused, concise and clearly worded, open-ended. They were developed covering how and why the requirements for Trustworthy AI would be applicable in clinical trials, and also in the case of widely used more innovative information technologies in conducting CTs (fig. 1).

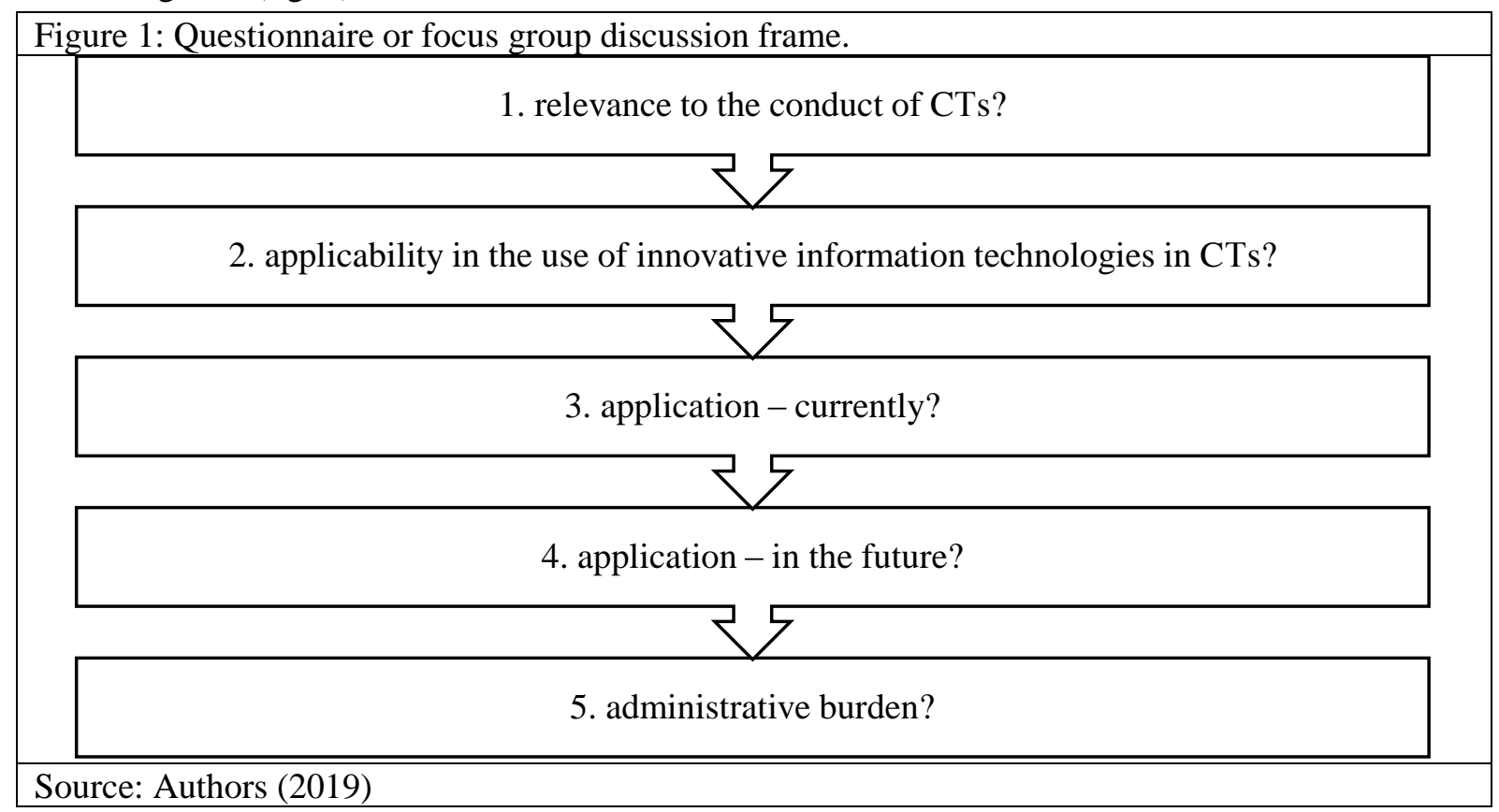

The concrete wording of the questions was concerning the seven requirements for Trustworthy AI, in addition to the development, implementation and use of innovative information technology systems in CTs:

1. Are they relevant in clinical trials as a whole?

2. Would they be applicable to the use of innovative information technology as AI in CTs?

3. Are you currently applying part or the entire proposed list?

4. In the future, would you apply some or the entire proposed list?

5. Is the administrative burden of applying the requirements justified by the effect?

Focus group discussions have proven to be an effective way of gathering information on the implementation of the Trustworthy AI Guidelines in the case of the introduction of innovative information technologies into CTs and the ethical issues that arise in this case.

\section{Results}

The application of innovative information technologies in the field of CTs, is a catalyst for accelerating and improving the CTs' efficiency. Both the presentation, used as the focus group discussion frame, and the questionnaire results agree.

According to the discussion results the application of the seven requirements for trustworthy $\mathrm{AI}$ are relevant in the conduct of CTs, as a whole. CTs planning and conducting improve by the use of innovative information systems. Additionally, the seven requirements are applicable and should be taken into account.

It is worth mentioning the second question outcome, about the use of AI in CTs. The experts' position is that the proposed checklist for trustworthy AI is a very comprehensive framework for application in the CTs.

Even that most of the experts believe that innovative information technologies (e.g. blockchain and the Internet of Things) are being used at present; they still believe that this remains mainly with scientific and academic interest.

The interesting point in the fourth question is the fact that the ethical aspects and challenges are of the utmost importance and compliance in the conduct of CTs. Particular attention should be paid in cases where more vulnerable groups are involved and affected. 
The participants agree that the administrative burden is rather during the projects launching phase, as conducting CTs, but when the project is gaining momentum, it significantly reduces and in practice the effect far exceeds the resources invested.

\section{Conclusion}

The introduction of innovative information technologies in the conduct of clinical trials poses new challenges regarding compliance with ethical principles and norms. The proposed framework for trustworthy AI can be used as a basis for research and ethical compliance when conducting clinical trials using innovative information technologies. It is essential now more than ever to accelerate CTs by applying innovative information technologies and minimize the time to the market, but always considering the ethical issues and the safety of the patient. The focus group methodology used to obtain information from experts about the ethical aspects rise when innovative information technologies like artificial intelligence and big data analytics in clinical trials. The experts' feedback was positive regarding the usage of the proposed guidelines for trustworthy AI, as evaluation tool for the particular case of innovative information technologies use in clinical trials.

\section{References}

Artificial Intelligence, High-Level Expert Group on (AI HLEG), (2018). Ethics Guidelines for Trustworthy AI. Retrieved from https://ec.europa.eu/futurium/en/ai-alliance-consultation/guidelines\#Top

Apressyan R. et. al., (2008). Advisory Expert Committee for the Teaching of Ethics, UNESCO, United Nations Educational, Scientific and Cultural Organization, Bioethics Core Curriculum. Retrieved from http://www.unesco-chairbioethics.org/?mbt_book=bioethics-core-curriculum

Beauchamp L., and Childress F., (2013), Principles of Biomedical Ethics, 7th Edition, Oxford University Press, ISBN: 978-019-992458-5

Harrer S., Shah P., Antony B., Hu J. (Harrer et al, 2019). Artificial Intelligence for Clinical Trial Design. Open Access Published: July 17, 2019. DOI:https://doi.org/10.1016/j.tips.2019.05.005. Retrieved from https://www.cell.com/trends/pharmacological-sciences/fulltext/S0165-6147(19)30130-0

Patias I. and Georgiev V., (2017). "Mobile Medical Applications: From Cloud-Oriented To Cloud Ready". MCIS 2017 Proceedings. 6. Retrieved from https://aisel.aisnet.org/mcis2017/6

UNESCO, (2005). Universal Declaration on Bioethics and Human Rights. The General Conference of UNESCO. Retrieved from http://portal.unesco.org/en/ev.php-URL_ID=31058\&URL_DO=DO_TOPIC\&URL_SECTION=201.html

Vodenitcharova A., and Popova K. (2019). Medical Ethics. FPH Press, Sofia. ISBN 978-619-7452-13-6.

WHO, (2020). Clinical Trials - International Registry Platform (ICTRP), World Health Organization, Clinical Trial. Retrieved from https://www.who.int/ictrp/glossary/en/ 